American Journal of Applied Sciences 4 (12): 1024-1028, 2007

ISSN 1546-9239

(C) 2007 Science Publications

\title{
Optical Properties and Kinetic Behavior of Chlorine in Pure Water and Swimming Pool Water using Surface Plasmon Resonance Technique
}

\author{
W.Y.W. Yusmawati and W. Mahmood Mat Yunus \\ Applied Optics Laboratory, Department of Physics, Faculty of Science, Universiti Putra Malaysia, \\ 43400 UPM Serdang, Selangor, Malaysia
}

\begin{abstract}
An optical sensor based on surface plasmon resonance phenomenon for detection of chlorine in pure water and swimming pool water is presented. The measurement was carried out at room temperature using Kretschmann surface plasmon resonance technique. When the air medium outside the metal film is changed to chlorine solution, the resonance angle shifted to the higher value. The shift of resonance angle $(\Delta \theta)$ increases linearly with chlorine concentration in which the detection limit and sensor sensitivity could be quantified. In this work, the sensitivity of the detection was estimated to be $0.11 \% \mathrm{ppm}$ and $0.12 \% \mathrm{ppm}$ for Calcium Hypochlorite (G70) and Trichloroisocyanuric Acid (G90), respectively. The detection limit of this sensor is better than $0.1 \mathrm{ppm}$ for chlorine samples and capable to monitor the chlorine concentration in swimming pool. We observed that the shift in the resonance angle $(\Delta \theta)$ decreases with time due to reducing amount of chlorine in the solution. Result from real-time measurement of swimming pool water was compared with simulation result carried out in the laboratory. Both have shown that the resonance angle decreases with time due to releasing of chlorine gas to the atmosphere.
\end{abstract}

Key words: Calcium hypochlorite, trichloroisocyanuric acid, detection limit, sensor sensitivity, swimming pool water

\section{INTRODUCTION}

Swimming pool water needs to be disinfected to prevent swimmer from being infected by microbial pathogen ${ }^{[1]}$. The main element for swimming pool disinfections is chlorine. It is important to ensure the water in the pool is safe and free from algae. By maintaining a free chlorine residual of not less than 1.5 ppm, algae infestation can be prevented. It is therefore paramount to maintain free residual chlorine at a level adequate enough to disinfect bacteria and virus that may be brought into the swimming pool water by swimmer, air dust, rain and other sources.

Surface plasmon resonance (SPR) spectroscopy is a surface-sensitive technique that has been used to characterize the thickness and/or index of refraction of dielectric medium at noble metal surface. For the last decade, surface plasmon resonance sensors have been extensively studied ${ }^{[2]}$. Surface plasmon resonance technique has emerged as a powerful technique for a variety of chemical and biological sensor applications. In the 1983, Liedberg et al. ${ }^{[3]}$ first demonstrated the exploitation of SPR as chemical sensing.

SPR is an optical process in which light satisfying a resonance condition excites a charge-density wave propagating along the interface between a metal and dielectric material by monochromatic and p-polarized light beam. The intensity of the reflected light is reduced at a specific incident angle producing a sharp shadow (called surface plasmon resonance) due to the resonance energy occurs between the incident beam and surface plasmon wave.

SPR is regarded as a simple optical technique for surface and interfacial studies ${ }^{[4]}$ and shows the great potential for investigating biomolecules. SPR has been used to study the refractive index of liquid measurement ${ }^{[5,6]}$, pesticide detection ${ }^{[7]}$ and SPR also can be regarded as a significantly tool for analyzing saccharides where saccharides solution commonly has a high refractive index ${ }^{[8]}$. In this paper, we report the surface plasmon resonance technique as an effective optical sensor for monitoring the chlorine concentration level in swimming pool.

\section{MATERIALS AND METHODS}

The surface plasmon resonance measurement has been carried out by measuring the reflected $\mathrm{He}-\mathrm{Ne}$ laser

Corresponding Author: W.Y.W. Yusmawati, Applied Optics Laboratory, Department of Physics, Faculty of Science, Universiti Putra Malaysia, 43400 UPM Serdang, Selangor, Malaysia 


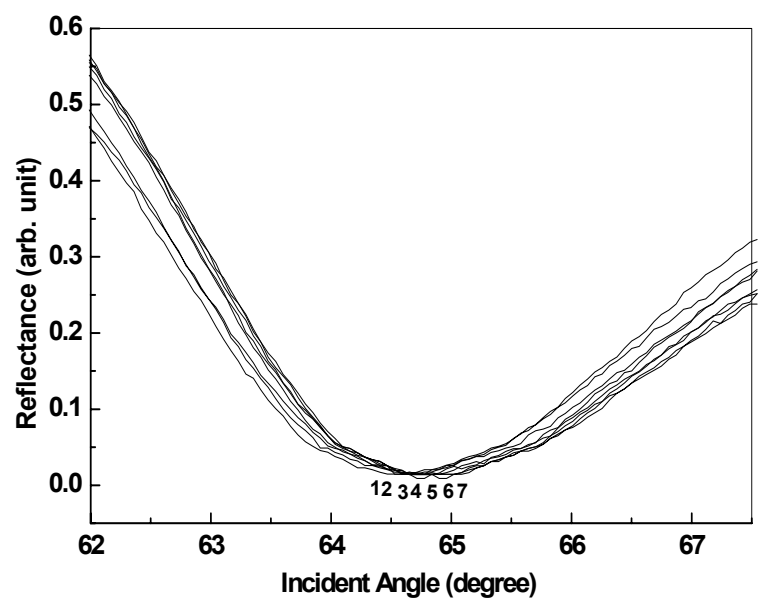

Fig. 1: Optical reflectance as a function of incidence angle for Calcium Hypochlorite at different concentration. [1: Distilled water; $2: 1 \mathrm{ppm} ; 3$ : 2 ppm 4: 3 ppm; 5: 4 ppm; 6: 5 ppm; 7: 6 ppm]

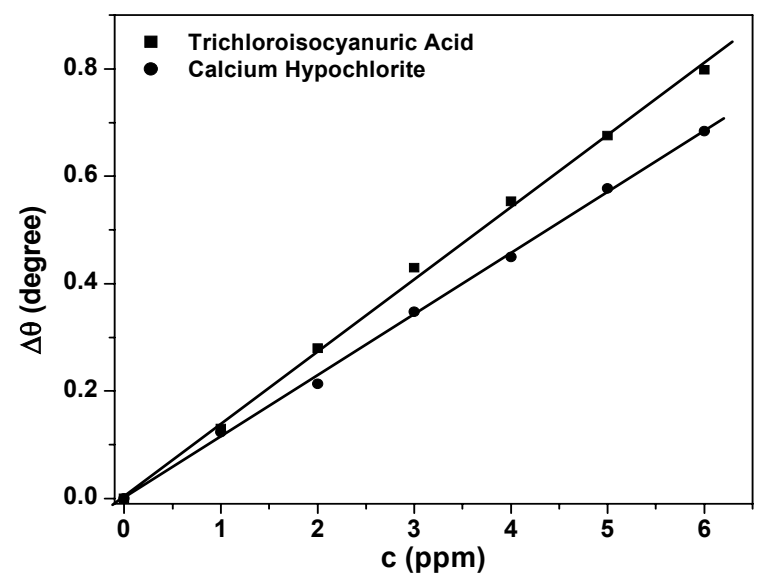

Fig. 2: The shift of resonance angle versus chlorine concentration (ppm)

beam $(632.8 \mathrm{~nm}, 5 \mathrm{~mW})$ as a function of incident angle. The detail experimental setup has been discussed in our previous paper ${ }^{[9]}$. The sample of Calcium Hypochlorite (G70) solution was prepared by systematically adding right amounts of G70 to distilled water to produce solution concentrations of 1 to $6 \mathrm{ppm}$. Similar procedure was applied to prepare the Trichloroisocyanuric Acid (G90) solutions. Both, G70 and G90 solutions were used as chlorinated agent in our measurements. For real application of surface plasmon optical sensor, the measurements were carried out on the swimming pool samples collected from 8.20 am to $5.20 \mathrm{pm}$ at Glory Resort swimming pool, Port Dickson, Negeri Sembilan.

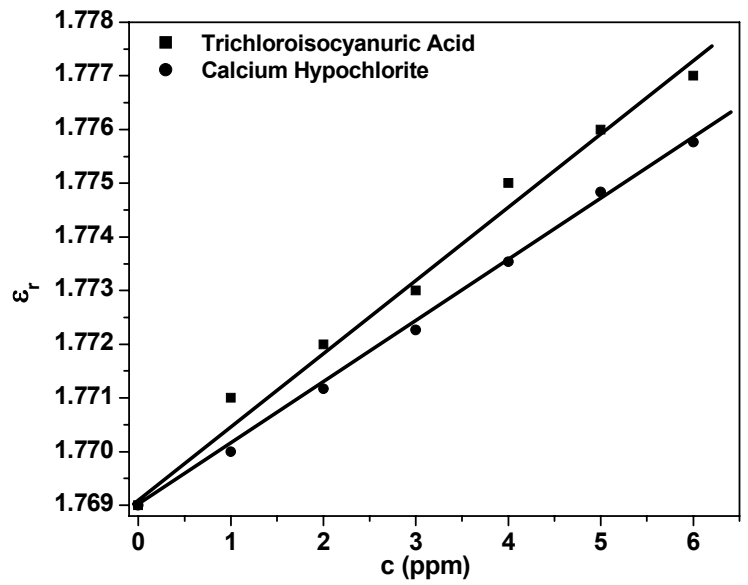

Fig. 3: The real part of dielectric constant, $\varepsilon_{\mathrm{r}}$ as a function of chlorine concentration

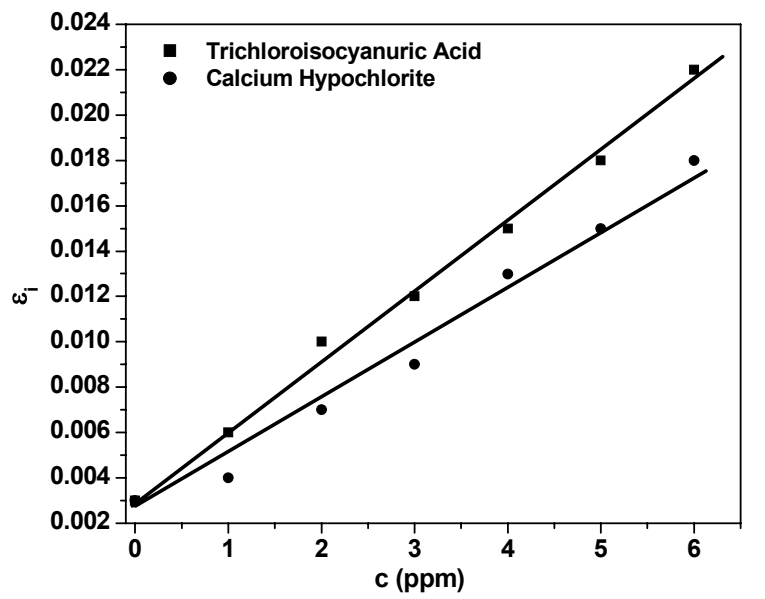

Fig. 4: The imaginary part of dielectric constant, $\varepsilon_{\mathrm{i}}$ as a function of chlorine concentration

\section{RESULTS AND DISCUSSION}

In this work, we used Calcium Hypochlorite (G70) and Trichloroisocyanuric Acid (G90) as a chlorine source. G70 was chosen because this chemical is a multi-functional sanitizer with utility in many industrial applications such as drinking water, beverage plants, canneries, fisheries, fruit and vegetable washing. It quickly forms hypochlorous acid (the killing form of chlorine) in water. On the other hand, the G90 is used for stabilized chlorine ion and solubility for pool water sanitization.

The concentration was chosen in the range of (1-6) ppm which is the same as the one used in swimming pool. For each sample, when the solutions of different concentrations (ppm) of the reagent were used, the angle shift has moved to the higher angle (Fig. 1). The 
shifts of resonance angle $(\Delta \theta)$ is linearly proportional to the concentration of Calcium Hypochlorite (G70) and Trichloroisocyanuric Acid (G90), as shown in Fig. 2. Since the straight lines passes through the origin, it is clear that the measurement of $\Delta \theta$ at lower concentrations of these reagents can be determined by this method. In the present work, the detection limit of this sensor was estimated to be better than $0.1 \mathrm{ppm}$ for both G70 and G90. Since the resolution of rotation angle was $0.001^{\circ}$, the slope of the straight lines which reflects to the sensitivity of the detection was estimated to be $0.114^{\circ} / \mathrm{ppm}$ and $0.120^{\circ} / \mathrm{ppm}$ for G70 and G90, respectively. For both of samples, the linear regression coefficient was 0.999 .

The value of real and imaginary part of dielectric constants, $\varepsilon_{\mathrm{r}}$ and $\varepsilon_{\mathrm{i}}$ of two chlorine solutions were obtained by fitting the experimental data to the Fresnel equation $^{[10,11]}$. Figure 3 and 4 shows the value of real and imaginary part of dielectric constants, $\varepsilon_{\mathrm{r}}$ and $\varepsilon_{\mathrm{i}}$ as a function of chlorine concentration. It shows that the values of $\varepsilon_{\mathrm{r}}$ and $\varepsilon_{\mathrm{i}}$ are linearly proportional to the chlorine concentration.

The kinetic behaviour of the chlorine sample was examined to monitor the self-assembling process on the metal surface in real time. Figure 5 shows the time dependence of the variation of the shift in resonance angle for G70 and G90 samples. The shift in resonance angle was observed to decrease with time and this may be due to the chlorine gas released to the atmosphere.

The experimental data in Fig. 5 are fitted to the empirical equation as:

$\theta(t)=\theta_{0}+A_{0} \exp \left[-\frac{K}{c} t\right]$

where $\theta_{0}$ and $A_{0}$ are constants, $K$ is kinetic constant and $c$ is the concentration of sample solution. The parameters obtained from this measurement are 0.085 and 0.094 for G70 and G90 samples, respectively.

For real application, we used our surface plasmon resonance sensor to monitor chlorine concentration in swimming pool water. Sample collection was done at every $90 \mathrm{~min}$ from 8.20 am to $5.20 \mathrm{pm}$ at Glory Resort swimming pool, Port Dickson where both G70 and G90 were used as chlorinated agents. Figure 6 shows the SPR shift of resonance angle as a function of sample collection time. We believed that the shift in resonance angle was due to changes of chlorine concentration in swimming pool water. The resonance angle shift decreases with time from 8.20 am to 9.50 am and followed by a constant value from 9.50 am to $1.00 \mathrm{pm}$. From $1.00 \mathrm{pm}$ to $5.20 \mathrm{pm}$, the resonance angles

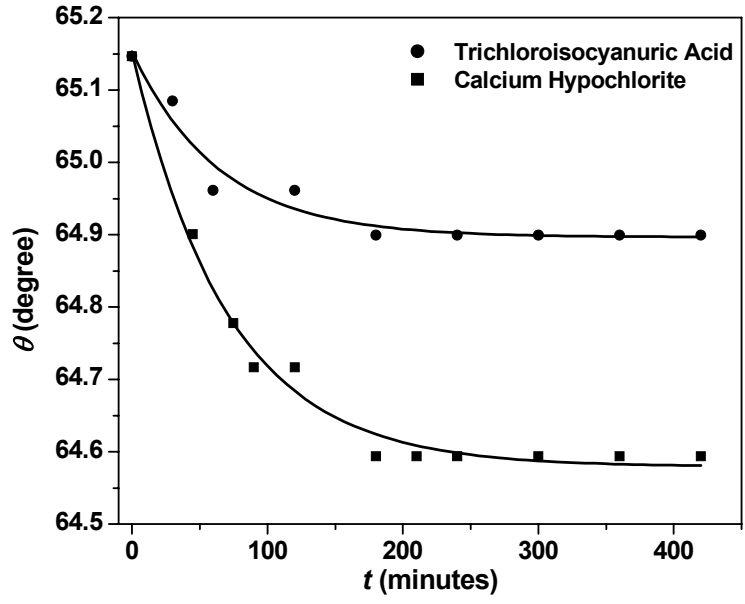

Fig. 5: The resonance angle shift versus time for two types of chlorine sample at $6 \mathrm{ppm}$

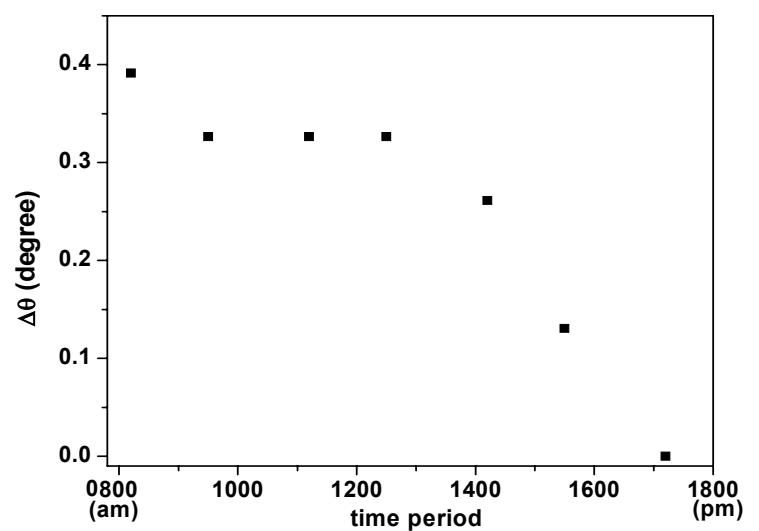

Fig. 6: The SPR shift of resonance angle as a function of time collected

decreases dramatically due to the more chlorine gas released to the atmosphere.

At $8.20 \mathrm{am}$, the concentration of both chlorine (G70 and G90) in swimming pool is the highest that records the biggest resonance angle shift. Then the concentration decreases as the ultraviolet rays of sunlight decompose chlorine and thus G90 and G70 evaporate from the swimming pool as the time goes by. Hence, the water sample collected at $5.20 \mathrm{pm}$ gives the smallest shift in resonance angle.

Ultraviolet light degrades chlorine by a photochemical reaction:

$2 \mathrm{Cl}_{2}+2 \mathrm{H}_{2} \mathrm{O} \stackrel{\mathrm{UV}}{\rightarrow} 4 \mathrm{HCl}+\mathrm{O}_{2}$

When the chlorine dissolves in water it disproportionate into hydrochloric acid $(\mathrm{HCl})$ and hypochlorous acid $(\mathrm{HClO})$. 


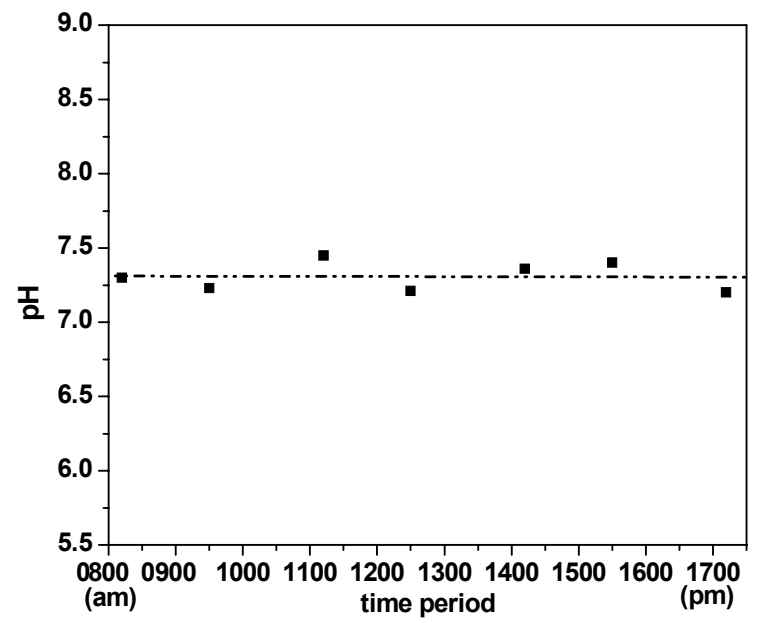

Fig. 7: The $\mathrm{pH}$ value as a function of time collected

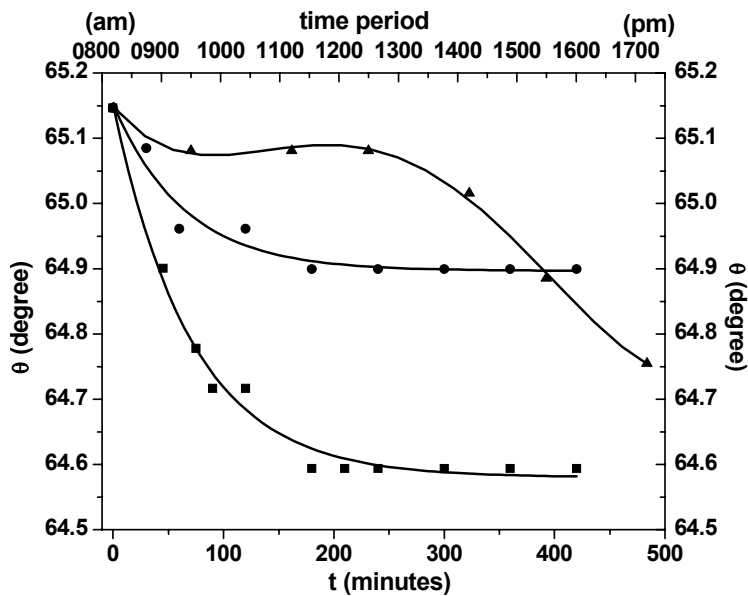

Fig. 8: The resonance angle versus time circle: G90; square: G70 and triangle: swimming pool water, respectively

$\mathrm{Cl}_{2}+\mathrm{H}_{2} \mathrm{O} \leftrightharpoons \mathrm{HClO}+\mathrm{H}^{+}+\mathrm{Cl}^{-}$

The hypochlorous acid ( $\mathrm{HClO})$ is a weak acid and it partially dissociates into hydrogen ions $\left(\mathrm{H}^{+}\right)$and hypochlorous ions $\left(\mathrm{OCl}^{-}\right)$

$\mathrm{HClO} \leftrightharpoons \mathrm{H}^{+}+\mathrm{ClO}^{-}$

Hypochlorous acid is the principle water disinfectant. It is widely used to kill bacteria and algae and an affective biocide since $\mathrm{ClO}^{-}$is strong oxidizing agent ${ }^{[12]}$. The Hypochlorous acid remnant is called free residual chlorine. Thus, chlorine as $\mathrm{HClO}$ is needed for sanitation and chlorine as $\mathrm{ClO}^{-}$is needed for oxidization.

Both of these reactions are influenced by the $\mathrm{pH}$ of the swimming pool water. Figure 7 shows the $\mathrm{pH}$ value as a function of collection time. In the swimming pool water treatment, $\mathrm{pH}$ is the most important part of pool water chemistry. We observed that the $\mathrm{pH}$ value of the swimming pool water is between 7.2 and 7.5 and agreed with the reference value $(6.5-7.6)^{[13]}$, which needs to be maintained for maximizing the sanitizing effect of the chlorine.

Finally, the kinetic behavior of the system was examined. Figure 8 shows the comparison of the resonance angle as a function of time between chlorine (G70 and G90) that was prepared in laboratory and in swimming pool water. The resonance angle shifts to lower value with increasing time for the entire chlorine sample. The shift in incidence angle was observed to decrease with time due to the combination of the chlorine ion with the impurities in the water that reduce the chlorine concentration and more chlorine gas released to the atmosphere.

\section{CONCLUSION}

A simple optical sensor based on Kretschmann surface plasmon resonance has been tested by measuring the chlorine concentration in water. The sensitivity of the detection was estimated to be $0.11 \% \mathrm{ppm}$ and $0.12 \% \mathrm{ppm}$ for Calcium Hypochlorite (G70) and Trichloroisocyanuric Acid (G90), respectively. The detection limit of this sensor is better than $0.1 \mathrm{ppm}$ for chlorine samples and capable to monitor the chlorine concentration in swimming pool. The kinetic behaviour was also examined by monitoring the self-assembling process on the metal surface in real time. The shift in incidence angle was observed decreasing with time due to decreasing chlorine concentration in the swimming pool water.

\section{ACKNOWLEDGEMENT}

The authors would like to thank the Malaysian Government for financial support through IRPA program. The laboratory facilities provided by the Department of Physics, UPM are also acknowledged.

\section{REFERENCES}

1. Powick, P.E.J., 1989. Swimming pool-brief outline of water treatment and management. Water Sci. Technol., 21: 151.

2. Homola, J., S. Yee and G. Gauglitz, 1999. Surface plasmon resonance sensors: review. Sens. Actuators, B 544: 3.

3. Liedberg, B., C. Nylander and I. Lundström, 1983. Surface Plasmon resonance for gas detection and biosensing. Sens. Actuators, B4: 299. 
4. Salamon, Z, H.A. Macleod, H.A. and Tollin, G., 1997. Surface plasmon resonance spectroscopy as a tool for investigating the biological and biophysical properties of membrane protein system I: Theoretical principles. Biochem. Biophys. Acta, 1331: 131

5. Matsubara, K., S. Kawata and S. Minami, 1988. Optical chemical sensor based on surface plasmon measurement. Appl. Opt., 27: 1160.

6. Kano, H. and S. Kawata, 1995. Grating-coupled surface plasmon for measuring the refractive index of a liquid sample. Jpn. J. Appl. Phys., 34: 331.

7. Chegel, .I., Y.M. Shirshov, E.V. Piletskaya and S.A. Piletsky, 1998. Surface plasmon resonance sensor for pesticide detection. Sens. Actuators, B 48: 456.

8. Zhen, W. and C. Yi, 2001. Analysis of mono- and oligosaccharide by multiwavelength surface plasmon resonance (SPR) spectroscopy. Carbohydrate Res., 332: 209.
9. Yusmawati, W.Y.W., H.P. Chuah and M.Y.W. Mahmood, 2007. Optical properties and sugar content of commercial carbonated drinks using surface plasmon resonance. Am. J. Appl. Sci., 4: 1.

10. Sincerbox, G.T. and J.C. Gordon II, 1981. Small fast large-aperture light modulator using attenuated total reflection. J. Appl. Opt., 20: 1491.

11. Lévesque, L., B.E. Paton and S.H. Payne, 1994. Precise thickness and refractive index determination of polymide film using attenuated total reflection. J. Appl. Opt., 33: 8036.

12. Pinto, G. and B. Rohrig, 2003. Use of Chloroisocyanuarates for disinfection of water: Application of miscellaneous general chemistry topics. J. Chem. Edu., 80: 41.

13. Borgmann-Strahsen, R., 2003. Comparative assessment of different biocides in swimming pool water. Intl. Biodeterioration \& Biodegradation, 51: 291. 